\title{
Impacts of Rapid Recanalization and Collateral Circulation on Clinical Outcome after Intraarterial Thrombolysis
}

\author{
Hye Seon Jeong, ${ }^{a, b}$ Hyon-Jo Kwon, ${ }^{a, c}$ Hee-Jung Song, ${ }^{a, b}$ Hyeon-Song Koh, ${ }^{a, c}$ Yong Soo Kim, ${ }^{\text {a,b }}$ \\ Ju Hun Lee, ${ }^{\mathrm{a}, \mathrm{b}}$ Jee Eun Shin, ${ }^{\mathrm{d}}$ Suk Hoon Lee, ${ }^{\mathrm{d}}$ Jei Kim ${ }^{\mathrm{a}, \mathrm{b}}$ \\ ${ }^{a}$ Regional Cerebrovascular Center, Hospital and School of Medicine, chungnam National University, Daejeon, Korea \\ bepartments of Neurology, Hospital and School of Medicine, Daejeon, Korea \\ 'Department of Neurosurgery, Hospital and School of Medicine, Daejeon, Korea \\ ${ }^{\mathrm{d}}$ Department of Information and Statistics, College of Natural Science, Chungnam National University, Daejeon, Korea
}

Background and Purpose Rapid recanalization might improve clinical outcomes after intraarterial thrombolysis (IAT) for acute ischemic stroke patients with collateral circulation. We determined whether rapid recanalization and collateral circulation affect clinical outcomes after IAT.

Methods We retrospectively evaluated the clinical and radiological data of 134 consecutive patients who underwent IAT for intracranial artery occlusion. The interval from symptom onset to recanalization after IAT (onset-to-recanalization time) as an estimate of the probability of good clinical outcome (modified Rankin scale 0-2) was calculated in patients with collateral circulation in the ischemic hemisphere, which was rated poor (0/1 American Society of Interventional and Therapeutic Neuroradiology criteria) or good (2-4). Changes in National Institute of Health Stroke Scale (NHISS) score before and after IAT and modified Rankins scale scores 3 months after discharge were compared with respect to onset-to-recanalization time. Results In patients with good collateral circulation, the estimated onset-to-recanalization time for a 0.5 probability of a good clinical outcome was 347 minutes; with poor collateral circulation, it was 172 minutes for a 0.2 probability of good clinical outcome. Outcome analyses according to onset-to-recanalization time showed patients recanalized $<6$ hours had lower NHISS scores $(<4.5,4.5-6$, $>6$ hours of onset-to-recanalization time, and non-recanalization: 5.1, 6.9, 11.9, and 19.8, respectively) at discharge and higher percentages of good clinical outcome $(69 \%, 66.7 \%, 21.9 \%$, and 0\%, respectively) 3 months after IAT.

Conclusions The time window to expect a high probability of a good clinical outcome after IAT is highly dependent on the collateral circulation.

Keywords Ischemic stroke; Thrombolysis; Collateral circulation; Recanalization; Clinical outcome

\section{Introduction}

Successful arterial recanalization is critical for improving immediate and long-term outcomes after thrombolytic therapy. ${ }^{1,2}$
Correspondence: Jei Kim Department of Neurology, Chungnam National University Hospital, 282 Moonhwa-ro, Joong-gu, Daejeon 301-721, Korea Tel: +82-42-280-7805 Fax: +82-42-252-8654 E-mail: jeikim@cnu.ac.kr

Received: October 6, 2014 Revised: January 16, 2015 Accepted: January 16, 2015

The authors have no financial conflicts of interest. 
nous thrombolysis (IVT) ${ }^{6-8}$ In addition to the time from symptom onset to recanalization (onset-to-recanalization time), the presence of collateral circulation is important for the achievement of good clinical outcomes after IAT, ${ }^{9,10}$ The onset-to-recanalization time window to achieve good clinical outcomes may be largely dependent on the degree of collateral circulation.

Therefore, this study investigated whether the degree of collateral circulation influences onset-to-recanalization time in association with good outcomes after IAT. We also determined the predictive powers of onset-to-recanalization time for good clinical outcomes on the basis of collateral circulation development in stroke patients who successfully underwent recanalization after IAT.

\section{Methods}

\section{Patients}

We retrospectively reviewed the clinical data of 4,028 patients with cerebral infarction admitted to Chungnam National University Hospital between January 2007 and February 2013. Then, 283 patients who underwent recanalization therapy, including 118, 74, and 91 who underwent IVT only, IVT-IAT, and IAT only, respectively, were selected. Finally, 134 consecutive patients who underwent IVT-IAT or IAT who met the following criteria were included: (1) age $>18$ years, (2) arrival at emergency room (ER) within 6 hours of symptom onset, (3) National Institutes of Health Stroke Scale (NIHSS) score $\geq 4,{ }^{11}$ and (4) occlusion of a unilateral middle cerebral artery (MCA) and/or terminal internal carotid artery confirmed by cerebral angiography. The study protocol was approved by the Institutional Review Board of Chungnam National University Hospital; the need for informed consent from the patients was waived because of the retrospective nature of this study.

\section{Critical pathways for intraarterial thrombolysis}

IAT was performed as either bridging therapy after IVT or primary therapy as per the critical pathway established at the Regional Cerebrovascular Center of Chungnam National University Hospital. ${ }^{12}$ Patients who arrived at the ER within 3 hours of symptom onset with no hemorrhage or significant low density on initial computed tomography (i.e., less than one-third of the MCA territory) were administered $0.9 \mathrm{mg} / \mathrm{kg}$ recombinant tissue plasminogen activator ( $\mathrm{tPA}$ ). If the patient exhibited no clinical improvement after IVT and there were image mismatches between diffusion- and perfusion-weighted images on magnetic resonance imaging (MRI), IAT was performed as bridging therapy. Patients who arrived 3-6 hours after symptom onset were treated with IAT after confirming the absence of hemorrhage on initial computed tomography and the presence of diffusion-/ perfusion-weighted image mismatch on MRI.

\section{Intraarterial thrombolysis procedure}

From January 2007 to October 2010, the IAT procedures included aggressive mechanical clot disruption using a microcatheter and/or a J-shaped guidewire tip. ${ }^{13}$ From November 2010 to February 2013, we used stent-assisted thrombectomy with a retrievable stent (Solitaire AB, ev3, Covidien, Dublin, Ireland). ${ }^{12}$ If the recanalized artery became re-occluded after stent-assisted thrombectomy, balloon angioplasty and/or stenting were performed.

\section{Clinical and radiological evaluation}

Demographic characteristics, vascular risk factors (e.g., hypertension, diabetes, smoking, and atrial fibrillation), radiological findings, and clinical outcomes before and after IAT were recorded. Stroke severity was assessed according to the NIHSS score. The time of successful recanalization after IAT was determined according to the times from (1) symptom onset to ER arrival (onset-to-door time), (2) ER arrival to groin puncture for IAT (door-to-puncture time), and (3) onset-to-recanalization.

Recanalization status after IAT was classified according to the Thrombolysis in Cerebral Infarction (TICI) grade; ${ }^{14}$ successful recanalization was defined as TICI grades $2 / 3 .{ }^{15}$ The collateral circulation status in the occluded hemisphere was determined by pre-IAT angiography. The collateral circulation extent was classified as poor (grades 0/1) or good (grades 2-4) according to the American Society of Interventional and Therapeutic Neuroradiology (ASITN) criteria. ${ }^{14,16}$ Symptomatic intracerebral hemorrhage (ICH) was defined as a $\geq 4$-point increase in the NIHSS score along with a large hematoma within 24 hours of IAT. ${ }^{17}$

Clinical improvement was assessed according to the changes in the NIHSS score immediately, 24 hours, and 3 days after IAT, and at discharge. Clinical outcomes were assessed according to the modified Rankin scale (mRS) score 3 months after discharge; an mRS score from 0 to 2 was considered a good clinical outcome.

\section{Statistical analysis}

To determine which factors are associated with good clinical outcomes after IAT, clinical and radiological variables, including age, risk factors, occlusion site, initial NIHSS score, intravenous tPA use before IAT, collateral circulation status, IAT method, procedure time, onset-to-recanalization time, recanalization grade, and the development of symptomatic ICH were analyzed using the $t$ test or $\chi^{2}$-test. Multiple logistic regression analysis was performed using variables with a $P$ value $<0.05$ in univariate analysis. 
Unadjusted fitted regression analysis was subsequently performed to determine whether onset-to-recanalization time and the presence of collateral circulation predict the probability of a good clinical outcome in patients with successful angiographic recanalization. First, a fitted straight line was calculated using the inverse regression method to estimate a calibration curve for a $0.1-0.8$ probability of a good clinical outcome. Then, the lower band from the lower endpoints of the $95 \%, 90 \%, 80 \%$, and $70 \%$ one-sided confidence intervals (CIs) was calculated to determine the true probability of a good clinical outcome for a given onset-to-recanalization time. The lower bound of each $\mathrm{CI}$ of onset-to-recanalization time was subsequently calculated for a specified probability of a good clinical outcome. Finally, the Xcoordinate of the intersection of the horizontal line parallel with the $\mathrm{X}$-axis was calculated at the specified probability and lower bound; it was designated the lower bound of the onset-to-recanalization time for the specified probability with a different CI. Inverse regression analyses between good clinical outcome and onset-to-recanalization time were performed with or without collateral circulation.

To determine if onset-to-recanalization time is associated with good clinical outcome, patients showing good collateral circulation (ASITN score 2-4) in the occluded hemisphere were categorized according to the fitted line and lower bound of 0.5 probability of a good clinical outcome (mRS score $0-2$ ).

Changes in clinical variables including the NIHSS score in each group were compared at different time points by repeated-measures ANOVA. Differences in the $\mathrm{mRS}$ scores between the groups 3 months after IAT were compared using Fisher's exact test.

All statistical analyses were performed using SPSS version 19.0 (SPSS Inc., Chicago, IL, USA). The level of significance was set at $P<0.05$.

\section{Results}

The clinical and radiological data of 134 consecutive patients ( 82 men; mean \pm SD age, $68.3 \pm 11.8$ years) who had undergone IAT for acute cerebral infarction are shown in Table 1. Intravenous tPA was administered before IAT in 64 patients (48\%). Before IAT, 84 (63\%) patients showed good collateral circulation in the ischemic hemisphere. Initial NIHSS score was significantly lower in patients with good collateral circulation than those with the poor collateral circulation $(11.6 \pm 3.6 \mathrm{vs}$. $13.2 \pm 3.4 ; P=0.013)$. After IAT, $111 / 134$ patients ( $83 \%)$ achieved successful recanalization (TICI grade $2 / 3$ ), including 42,37 , and 32 patients $<4.5,4.5-6$, and $>6$ hours from symptom onset, respectively. The mean onset-to-recanalization time was 308.5 \pm 94.8 minutes (range: 117-604 minutes).
Table 1. Patient demographics

\begin{tabular}{lc}
\hline Characteristics & $\mathrm{N}=134$ \\
\hline Age & $68.3 \pm 11.8$ \\
Sex (M:F) & $82: 52(61 \%: 39 \%)$ \\
Risk factors & \\
$\quad$ Hypertension & $66(49 \%)$ \\
Diabetes & $27(20 \%)$ \\
Smoking & $27(20 \%)$ \\
Atrial fibrillation & $78(58 \%)$ \\
Occluded artery & \\
TICA & $39(29 \%)$ \\
MCA & $95(71 \%)$ \\
$\quad$ M1 portion & $87(65 \%)$ \\
$\quad$ M2 portion & $8(6 \%)$ \\
Initial NIHSS score (median, interquartile range) & $10,13-15$ \\
Intravenous tPA before IAT & $64(48 \%)$ \\
Initial collateral circulation status & \\
Good (ASITN grade 2-4) & $84(63 \%)$ \\
Poor (ASITN grade 0-1) & $50(37 \%)$ \\
Symptom onset to ER arrival (min) & $130.8 \pm 93.0$ \\
ER arrival to groin puncture (min) & $91.0 \pm 26.8$ \\
Onset to groin puncture for IAT (min) & $210.5 \pm 65.1$ \\
Onset to recanalization time (min) & $308.5 \pm 94.8$ \\
Recanalization time & \\
< 4.5 hours & $42(31 \%)$ \\
4.5-6 hours & $37(28 \%)$ \\
> 6 hours & $32(24 \%)$ \\
No recanalization (TICI 0-1) & $23(17 \%)$ \\
\hline
\end{tabular}

Values are mean $\pm S D$ or $n(\%)$ unless indicated otherwise.

$\mathrm{M}$, male; $\mathrm{F}$, female; T-ICA, terminal internal carotid artery; MCA, middle cerebral artery; NIHSS, National Institutes of Health Stroke Scale; PPA, tissue plasminogen activator, ASITN, American Society of Interventional and Therapeutic Neuroradiology; ER, emergency room; IAT, intraarterial thrombolysis; TICI, Thrombolysis in Cerebral Infarction.

\section{Variables associated with clinical outcome after intraarterial thrombolysis}

Univariate analysis showed young age, MCA occlusion site, good collateral circulation, rapid onset-to-recanalization time, successful recanalization, and no symptomatic ICH were significantly associated with good clinical outcome at discharge (Table 2). Meanwhile, stepwise regression analysis of the abovementioned covariates indicated that the presence of good collateral circulation (odds ratio [OR]: 9.624, 95\% CI: 2.81932.864; $P<0.001$ ) and rapid onset-to-recanalization time (OR: $1.006,95 \%$ CI: 0.001-1.011; $P=0.019)$ were independently associated with good clinical outcome (Table 2).

\section{Relationship between onset-to-recanalization time} and good clinical outcome with respect to collateral circulation

The impact of collateral circulation on the relationship between onset-to-recanalization time and good clinical outcome is 
shown in Figure 1 and Supplementary Table I. In patients with good collateral circulation before IAT, the estimated onset-to-recanalization time for a 0.5 probability of a good clinical outcome was 347 minutes. The maximum onset-to-recanalization time to expect a 0.5 probability of a good clinical outcome (i.e., the lower bound of the $95 \%$ one-sided CIs of the probability) was 275 minutes. In patients with poor collateral circulation, the expected and maximum onset-to-recanalization times were dramatically shorter: that for a 0.2 probability of a good clinical outcome was 172 minutes. The maximum onset-to-recanalization time was 31 minutes if the confidence limit was lowered to $70 \%$.

\section{Clinical outcomes according to onset-to-recanalization time}

To determine the onset-to-recanalization time interval required for a 0.5 probability of a good clinical outcome in the presence of collateral circulation, patients were classified ac- cording to onset-to-recanalization times of $<4.5,4.5-6$, or $>6$ hours, or non-recanalization after IAT. Although the mean initial NIHSS score was similar among groups, improvements in the NIHSS score after IAT differed significantly. Patients recanalized within 4.5 or 6 hours (both $P<0.001$ ) had significantly lower mean NIHSS scores after IAT and at discharge than patients recanalized after $>6$ hours $(P=0.021)$ or those who did not undergo recanalization $(P=0.002)$ (Figure 2).

Sixty $(44.8 \%)$ of 134 patients achieved good clinical outcomes (mRS score 0-2) 3 months after IAT. After classifying the patients according to onset-to-recanalization time, the proportion of patients who achieved good clinical outcome 3 months after IAT was significantly greater in those recanalized in $<6$ hours than those recanalized $>6$ hours or who did not undergo recanalization $(P<0.001)$ (Figure 3$)$. In contrast, the proportion of patients with poor clinical outcomes ( $\mathrm{mRS}$ score 5-6) 3 months after IAT was significantly greater in patients re-

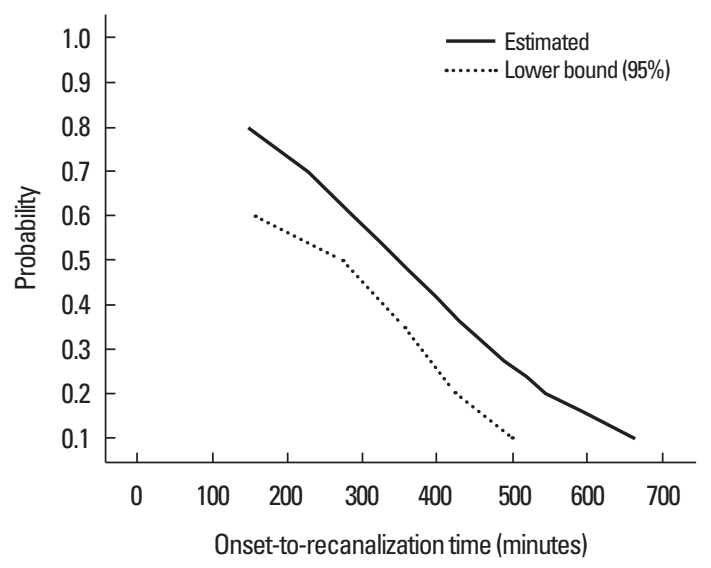

A

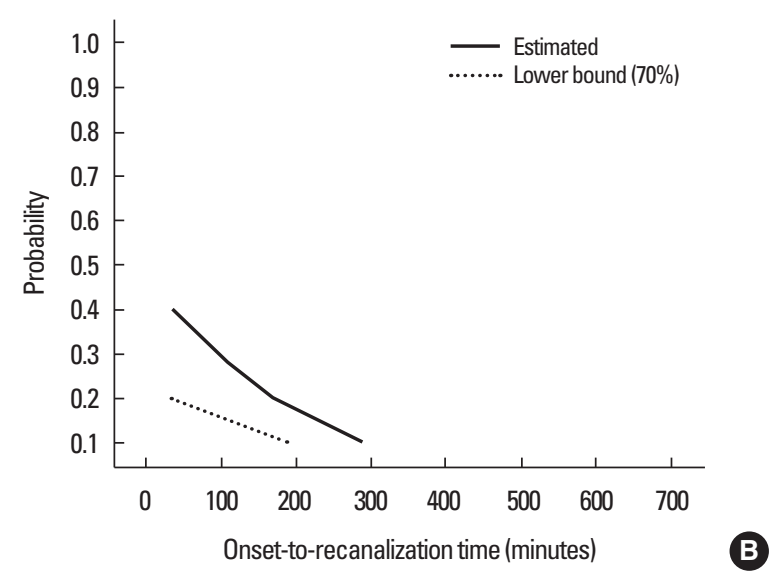

Figure 1. Estimated and lower bounds of onset-to-recanalization times with respect to the probability of a good clinical outcome according to (A) good and (B) poor collateral circulation status before intraarterial thrombolysis in successfully recanalized patients. Estimated, estimated onset-to-recanalization time for a specified probability of a good clinical outcome; lower bound, lower bound of the onset-to-recanalization time for specified probability of a good clinical outcome with 95\% and $70 \%$ confidence intervals in patients with good and poor collateral circulation, respectively.

Table 2. Univariate and multivariate analyses of the associations of clinical and radiological variables with good clinical outcome at discharge

\begin{tabular}{|c|c|c|c|c|c|c|}
\hline & \multicolumn{3}{|c|}{ Univariate analysis } & \multicolumn{3}{|c|}{ Multiple logistic regression } \\
\hline & $\mathrm{OR}$ & $95 \% \mathrm{Cl}$ & $P$ value & $\mathrm{OR}$ & $95 \% \mathrm{Cl}$ & $P$ value \\
\hline Age & 1.038 & $1.006-1.071$ & 0.021 & 1.022 & $0.982-1.063$ & 0.293 \\
\hline Occluded artery (MCA) & 2.818 & $1.172-6.777$ & 0.019 & 0.846 & $0.268-2.671$ & 0.776 \\
\hline Atrial fibrillation & 0.519 & $0.253-1.062$ & 0.073 & - & - & - \\
\hline Hypertension & 0.920 & $0.454-1.865$ & 0.817 & - & - & - \\
\hline Diabetes & 1.068 & $0.445-2.564$ & 0.883 & - & - & - \\
\hline Intravenous tPA before IAT & 0.587 & $0.288-1.197$ & 0.143 & - & - & - \\
\hline Good collateral circulation & 16.867 & $4.862-58.514$ & $<0.001$ & 9.624 & $2.819-32.864$ & $<0.001$ \\
\hline Recanalization time & 1.009 & $1.004-1.013$ & $<0.001$ & 1.006 & $1.001-1.011$ & 0.019 \\
\hline Successful recanalization (TICI 2/3) & 7.344 & 2.182-128.766 & 0.007 & 7.031 & $0.776-63.672$ & 0.083 \\
\hline Symptomatic ICH & 0.190 & $0.042-0.867$ & 0.032 & 0.201 & $0.036-1.119$ & 0.067 \\
\hline
\end{tabular}

IAT, intraarterial therapy; MCA, middle cerebral artery; tPA, tissue plasminogen activator; TICI, Thrombolysis in Cerebral Infarction; ICH, intracerebral hemorrhage; OR, odds ratio; $\mathrm{Cl}$, confidence interval. 


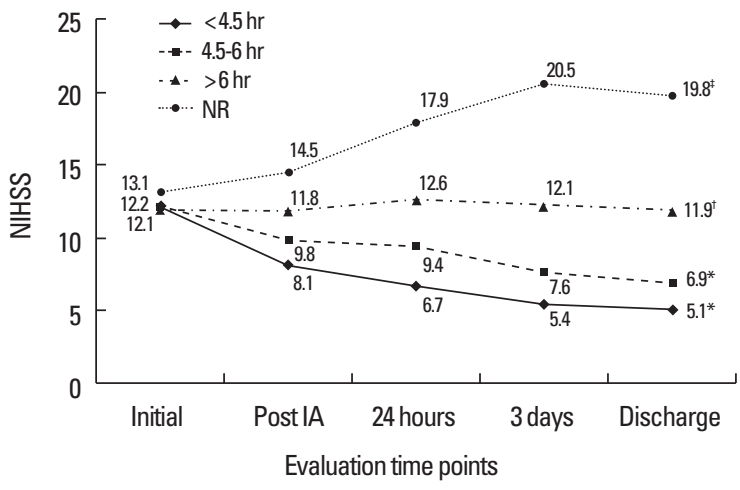

Figure 2. Changes in median National Institute of Health Stroke Scale (NIHSS) scores before and after intraarterial thrombolysis (IAT) and at discharge according to onset-to-recanalization time. Onset-to-recanalization time was categorized as $<4.5,4.5-6$, or $>6$ hours, or non-recanalization. ${ }^{*} P<0.001$, ${ }^{\dagger} P=0.021,{ }^{\ddagger} P=0.002$, changes in mean NIHSS score before and after IAT in each group.

canalized $>6$ hours and those who did not undergo recanalization than in those recanalized $<6$ hours $(P<0.001)$ (Figure 3); symptomatic ICH was also more frequent in the latter patients ( $<4.5,4.5-6,>6$ hours, and non-recanalization groups: $2.4 \%$, $2.7 \%, 18.8 \%$, and $13 \%$, respectively, $P=0.028$ ).

\section{Discussion}

This study investigated the impact of collateral circulation on the relationship between onset-to-recanalization time and clinical outcome in patients who have undergone IAT. The results demonstrate that the onset-to-recanalization time window for achieving good clinical outcome differs with respect to the degree of collateral circulation. When collateral circulation to the ischemic hemisphere is good, rapid recanalization ( $<6$ hours) results in a 0.5 probability of a good clinical outcome. In contrast, poor collateral circulation significantly decreases the probability of a good clinical outcome; in such cases, the probability of a good outcome is only 0.2 even with early recanalization within 172 minutes. Because collateral circulation to the ischemic hemisphere decreases infarct size and leads to good clinical outcomes in acute ischemia patients, ${ }^{10,18}$ identifying collateral circulation on initial angiography before IAT can aid decision making for the rapid execution of IAT and prediction of good clinical outcome.

A recent study reporting a significant relationship between rapid recanalization and good clinical outcome did not consider the presence of collateral circulation. ${ }^{19}$ There are other differences between the present and previous study worth mentioning. First, the mean onset-to-recanalization time was longer in the previous study than the present study (325 vs. 305 minutes,

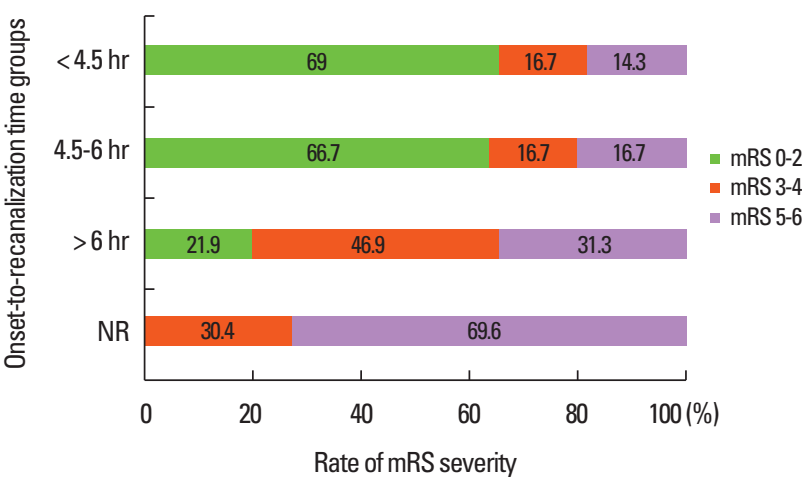

Figure 3. Modified Rankin scale (mRS) scores 3 months after intraarterial therapy (IAT) with respect to onset-to-recanalization. The proportions of patients with an mRS score from 0 to 2 (good outcome) 3 months after IAT differed significantly among groups $(P<0.001)$. NR, non-recanalization.

respectively). In the previous study, every 30 minutes of delay in onset-to-recanalization reduced the relative likelihood of a good clinical outcome by $12 \%$. Although similar results were obtained in the present study (Figure 1, Supplementary Table I), the probability of a good clinical outcome decreased even if the patients had good collaterals before the start of IAT. Second, the previous study included a greater proportion of patients with M2 occlusion (28\%) than the present study (8\%). Collateral development accompanied by M2 branch occlusion might differ from M1 trunk occlusion. Despite these differences, both studies demonstrate the importance of rapid recanalization for successful IAT and confirm shortening onset-to-recanalization time increases the probability of a good clinical outcome. ${ }^{1}$

Onset-to-recanalization time can also predict the probability of a good clinical outcome. In a previous study, patients who underwent IAT (33.6\%) were more likely to achieve a favorable outcome at 3 months than those who underwent IVT (24.9\%) for large artery occlusion. ${ }^{20}$ The recent application of mechanical devices for IAT has increased the recanalization rate. ${ }^{3,5} \mathrm{How}-$ ever, the favorable outcome rate for IAT has failed to meet expectations. ${ }^{6,7}$ Previous studies did not include onset-to-recanalization time as a variable when comparing the efficacy of IAT with IVT for acute ischemia patients. In the present study, $44 \%$ of patients achieved a good clinical outcome 3 months after IAT regardless of onset-to-recanalization time. The acute and long-term changes in clinical severity differed between patients recanalized in $\leq 6$ or $>6$ hours after IAT. In patients recanalized within 6 hours, the initial severity improved significantly after IAT. However, clinical severity did not improve in patients who were recanalized beyond 6 hours after IAT or those who did not achieve recanalization. More than $67 \%$ of patients recanalized within 6 hours could perform daily activities independently compared to $22 \%$ and $0 \%$ of patients recanalized beyond 
6 hours after IAT and those who did not achieve recanalization. An interval of 6 hours was previously defined as the time window for IAT. In the present study, an onset-to-recanalization time of 6 hours was also the therapeutic time window to achieve a good clinical outcome. Therefore, this cut-off could be important for the timely prediction of long-term clinical outcomes and appropriate selection of patients for IAT.

This study has some limitations should be noted. First, the retrospective single-center design limits the generalizability of the findings. And, although the results suggest an onset-to-recanalization time $<6$ hours can increase the probability of a good clinical outcome in IAT patients, unfortunately, only 59\% of patients achieved this. Therefore, better methods are required to reduce the onset-to-recanalization time of IAT for acute ischemic stroke patients. Future studies should focus on extending the onset-to-recanalization time window beyond 6 hours to improve clinical outcomes after IAT. To confirm the predictive values of the promptness of IAT and collateral circulation for good outcomes after IAT, criteria for selecting the optimal time that best predicts a good clinical outcome should be determined.

In summary, the time window to expect a high probability of a good clinical outcome after IAT is highly dependent on the collateral circulation. That is, rapid recanalization is an independent predictor of good clinical outcome after IAT, and a short onset-to-recanalization time increases the probability of a good clinical outcome. There is a 6-hour time window from symptom onset to recanalization when a good clinical outcome can be obtained after IAT in patients with acute large artery occlusion. Furthermore, the presence of collateral circulation to the ischemic hemisphere on initial angiography is an important predictor of good clinical outcome after successful recanalization by IAT.

\section{Conflict of interest}

The authors declare no conflicts of interest.

\section{References}

1. Khatri P, Abruzzo T, Yeatts SD, Nichols C, Broderick JP, Tomsick TA. Good clinical outcome after ischemic stroke with successful revascularization is time-dependent. Neurology 2009; 73:1066-1072.

2. Mazighi M, Meseguer E, Labreuche J, Serfaty JM, Laissy JP, Lavallée PC, et al. Dramatic recovery in acute ischemic stroke is associated with arterial recanalization grade and speed. Stroke 2012;43:2998-3002.
3. Castaño C, Dorado L, Guerrero C, Millán M, Gomis M, Perez de la Ossa N, et al. Mechanical thrombectomy with the Solitaire $\mathrm{AB}$ device in large artery occlusions of the anterior circulation: a pilot study. Stroke 2010;41:1836-1840.

4. Smith WS, Sung G, Starkman S, Saver JL, Kidwell CS, Gobin YP, et al. Safety and efficacy of mechanical embolectomy in acute ischemic stroke: results of the MERCI trial. Stroke 2005;36: 1432-1438.

5. Penumbra Pivotal Stroke Trial Investigators. The penumbra pivotal stroke trial: safety and effectiveness of a new generation of mechanical devices for clot removal in intracranial large vessel occlusive disease. Stroke 2009; 40:2761-2768.

6. Broderick JP, Palesch YY, Demchuk AM, Yeatts SD, Khatri P, Hill MD, et al. Endovascular therapy after intravenous t-PA versus t-PA alone for stroke. N Engl J Med 2013;368:893-903.

7. Ciccone A, Valvassori L, Nichelatti M, Sgoifo A, Ponzio M, Sterzi R, et al. Endovascular treatment for acute ischemic stroke. N Engl J Med 2013;368:904-913.

8. Kidwell CS, Jahan R, Gornbein J, Alger JR, Nenov V, Ajani Z, et al. A trial of imaging selection and endovascular treatment for ischemic stroke. N Engl J Med 2013;368:914-923.

9. Rha JH, Saver JL. The impact of recanalization on ischemic stroke outcome: a meta-analysis. Stroke 2007;38:967-973.

10. Marks MP, Lansberg MG, Mlynash M, Olivot JM, Straka M, Kemp S, et al. Effect of collateral blood flow on patients undergoing endovascular therapy for acute ischemic stroke. Stroke 2014;45:1035-1039.

11. Brott T, Adams HP Jr, Olinger CP, Marler JR, Barsan WG, Biller J, et al. Measurements of acute cerebral infarction: a clinical examination scale. Stroke 1989;20:864-870.

12. Jeong HS, Song HJ, Kim SB, Lee J, Kang CW, Koh HS, et al. A comparison of stent-assisted mechanical thrombectomy and conventional intra-arterial thrombolysis for acute cerebral infarction. J Clin Neurol 2013;9:91-96.

13. Sorimachi T, Fujii Y, Tsuchiya N, Nashimoto T, Harada A, Ito Y, et al. Recanalization by mechanical embolus disruption during intra-arterial thrombolysis in the carotid territory. Am J Neuroradiol 2004;25:1391-1402.

14. Higashida RT, Furlan AJ, Roberts H, Tomsick T, Connors B, Barr J, et al. Trial design and reporting standards for intra-arterial cerebral thrombolysis for acute ischemic stroke. Stroke 2003; 34:e109-137.

15. Roth C, Papanagiotou P, Behnke S, Walter S, Haass A, Becker $\mathrm{C}$, et al. Stent-assisted mechanical recanalization for treatment of acute intracerebral artery occlusions. Stroke 2010;41:25592567.

16. Bang OY, Saver JL, Buck BH, Alger JR, Starkman S, Ovbiagele $B$, et al. Impact of collateral flow on tissue fate in acute isch- 
aemic stroke. J Neurol Neurosurg Psychiatry 2008;79:625-629.

17. Khatri P, Wechsler LR, Broderick JP. Intracerebral hemorrhage associated with revascularization therapies. Stroke 2007;38: 431-440.

18. Christoforidis GA, Mohammad Y, Kehagias D, Avutu B, Slivka AP. Angiographic assessment of pial collaterals as a prognostic indicator following intra-arterial thrombolysis for acute ischemic stroke. Am J Neuroradiol 2005;26:1789-1797.

19. Khatri P, Yeatts SD, Mazighi M, Broderick JP, Liebeskind DS,
Demchuk AM, et al. Time to angiographic reperfusion and clinical outcome after acute ischaemic stroke: an analysis of data from the Interventional Management of Stroke (IMS III) phase 3 trial. Lancet Neurol 2014;13:567-574.

20. Mokin M, Kass-Hout T, Kass-Hout O, Dumont TM, Kan P, Snyder KV, et al. Intravenous thrombolysis and endovascular therapy for acute ischemic stroke with internal carotid artery occlusion: a systematic review of clinical outcomes. Stroke 2012;43: 2362-2368. 
Supplementary Table I. Probability of a good clinical outcome owing to technically successful angiographic recanalization according to collateral circulation status before intraarterial thrombolysis

\begin{tabular}{|c|c|c|c|c|c|c|c|c|c|c|}
\hline \multirow{4}{*}{$\begin{array}{l}\text { Probability of good } \\
\text { clinical outcome }\end{array}$} & \multicolumn{10}{|c|}{ Collateral circulation status } \\
\hline & \multicolumn{5}{|c|}{ Good } & \multicolumn{5}{|c|}{ Poor } \\
\hline & \multirow{2}{*}{ Estimated } & \multicolumn{4}{|c|}{ Confidence interval } & \multirow{2}{*}{ Estimated } & \multicolumn{4}{|c|}{ Confidence interval } \\
\hline & & $95 \%$ & $90 \%$ & $80 \%$ & $70 \%$ & & $95 \%$ & $90 \%$ & $80 \%$ & $70 \%$ \\
\hline 0.8 & 147.56 & - & - & - & 32.568 & - & - & - & - & - \\
\hline 0.7 & 225.28 & - & 57.107 & 126.801 & 156.131 & - & - & - & - & - \\
\hline 0.6 & 288.99 & 159.713 & 203.168 & 232.105 & 246.271 & - & - & - & - & - \\
\hline 0.5 & 347.46 & 274.959 & 288.513 & 301.996 & 310.497 & - & - & - & - & - \\
\hline 0.4 & 405.93 & 331.795 & 341.5 & 353.124 & 361.456 & 30.19 & - & - & - & - \\
\hline 0.3 & 469.64 & 377.426 & 387.87 & 401.212 & 411.251 & 93.90 & - & - & - & - \\
\hline 0.2 & 547.36 & 426.679 & 439.471 & 456.268 & 469.182 & 171.62 & - & - & - & 31.942 \\
\hline 0.1 & 664.29 & 496.487 & 513.641 & 536.497 & 554.274 & 288.55 & 9.165 & 94.807 & 156.037 & 187.416 \\
\hline
\end{tabular}

A good clinical outcome was defined as a modified Rankin scale score from 0-2. Collateral circulation was classified as poor (grade 0-1) or good (grades 2-4) according to the American Society of Interventional and Therapeutic Neuroradiology (ASITN) criteria. 